\title{
Erratum
}

\section{Erratum to "Iterative Methods for Variational Inequalities over the Intersection of the Fixed Points Set of a Nonexpansive Semigroup in Banach Spaces"}

\author{
Issa Mohamadi \\ Department of Mathematics, Islamic Azad University, Sanandaj Branch, Sanandaj 418, Kurdistan, Iran \\ Correspondence should be addressed to Issa Mohamadi, imohamadi@iausdj.ac.ir \\ Received 22 February 2011; Accepted 24 February 2011 \\ Copyright (C) 2011 Issa Mohamadi. This is an open access article distributed under the Creative \\ Commons Attribution License, which permits unrestricted use, distribution, and reproduction in \\ any medium, provided the original work is properly cited.
}

In my recent published paper [1] to prove Lemmas 3.1 and 5.1, an inequality involving the single-valued normalized duality mapping $J$ from $X$ into $2^{X^{*}}$ has been used that generally turns out there is no certainty about its accuracy. In this erratum we fix this problem by imposing additional assumptions in a way that the proofs of the main theorems do not change.

We recall that a uniformly smooth Banach space $X$ is $q$-uniformly smooth for $q>1$ if and only if there exists a constant $\beta_{q}>0$ such that, for all $x, y \in X$,

$$
\|x+y\|^{q} \leq\|x\|^{q}+q\|x\|^{q-2}\langle y, J(x)\rangle+2 \beta_{q}\|y\|^{q},
$$

for more details see [2]. Therefore, if $q=2$, then there exists a constant $\beta>0$ such that

$$
\|x+y\|^{2} \leq\|x\|^{2}+2\langle y, J(x)\rangle+2 \beta\|y\|^{2} .
$$

It is well known that Hilbert spaces, $l_{p}$ and $L_{p}$ for $p \geq 2$, are 2-uniformly smooth. 
Throughout the paper we suggest to impose one of the following conditions:

(a) the Banach space $X$ is 2-uniformly smooth;

(b) there exists a constant $\beta \in \mathbb{R}^{+}$for which $J$ satisfies the following inequality:

$$
\langle y, J(x+y)\rangle \leq\langle y, J(x)\rangle+\beta\|y\|^{2},
$$

for all $x, y \in X$.

Remark 1.1. If $J$ is $\beta$-Lipschitzian, then $J$ satisfies (3) and is norm-to-norm uniformly continues that suffices to guarantee that $X$ is 2-uniformly smooth. For more results concerning $\beta$-Lipschitzian normalized duality mapping see [3].

Note that since every uniformly smooth Banach space $X$ has a Gateaux differentiable norm and each nonempty, bounded, closed, and convex subset of $X$ has common fixed point property for nonexpansive mappings, we have $D\left(x_{n}\right) \cap C \neq \emptyset$ in [1]. So, when $X$ is 2-uniformly smooth, we can remove these two conditions from Theorems 3.2, 4.2, and 5.2 in [1].

Considering the above discussion to complete our paper, we reprove Lemmas 3.1 and 5.1 of [1] here with some little changes.

Lemma 3.1 (see [1]). Either let $X$ be a real Banach space, and let $J$ be the single-valued normalized duality mapping from $X$ into $2^{X^{*}}$ satisfing (3) or let $X$ be a 2-uniformly smooth real Banach space. Assume that $F: X \rightarrow X$ is $\eta$-strongly monotone and $\kappa$-Lipschitzian on $X$. Then

$$
\psi(x)=I(x)-\mu F(x)
$$

is a contraction on $X$ for every $\mu \in\left(0, \eta / \beta \kappa^{2}\right)$.

Proof. If $J$ satisfies (3), considering the inequality

$$
\|x+y\|^{2} \leq\|x\|^{2}+2\langle y, J(x+y)\rangle,
$$

for all $x, y \in X$, we have

$$
\begin{aligned}
\|\psi x-\psi y\|^{2} & \leq\|(I-\mu F) x-(I-\mu F) y\|^{2}=\|(x-y)+\mu(F y-F x)\|^{2} \\
& \leq\|x-y\|^{2}+2\langle\mu(F y-F x), J((x-y)+\mu(F y-F x))\rangle \\
& \leq\|x-y\|^{2}+2 \mu\langle F y-F x, J(x-y)\rangle+2 \beta \mu^{2}\langle F y-F x, J(F y-F x)\rangle \\
& \leq\|x-y\|^{2}-2 \mu\langle F x-F y, J(x-y)\rangle+2 \beta \mu^{2}\|F y-F x\|\|J(F y-F x)\| \\
& \leq\|x-y\|^{2}-2 \mu \eta\|x-y\|^{2}+2 \beta \mu^{2}\|F y-F x\|^{2} \\
& \leq\|x-y\|^{2}-2 \mu \eta\|x-y\|^{2}+2 \mu^{2} \beta \kappa^{2}\|x-y\|^{2} \\
& \leq\left(1-2 \mu \eta+2 \mu^{2} \beta \kappa^{2}\right)\|x-y\|^{2} .
\end{aligned}
$$


Clearly, the same inequality holds if $X$ is a 2-uniformly smooth real Banach space. Thus, we obtain

$$
\|\psi x-\psi y\| \leq \sqrt{1-2 \mu\left(\eta-\mu \beta \kappa^{2}\right)}\|x-y\| .
$$

With no loss of generality we can take $\beta \geq 1 / 2$; therefore, if $\mu \in\left(0, \eta / \beta \kappa^{2}\right)$, then we have $\sqrt{1-2 \mu\left(\eta-\mu \beta \kappa^{2}\right)} \in(0,1)$; that is, $\psi$ is a contraction, and the proof is complete. follows.

Also Lemma 5.1, which is easily proved in the same way as Lemma 3.1, will be as

Lemma 5.1 (see [1]). Either let $X$ be a real Banach space, and let $J$ be the single-valued normalized duality mapping from $X$ into $2^{X^{*}}$ satisfing (3), or let $X$ be a 2-uniformly smooth real Banach space. Assume that $F: X \rightarrow X$ is $\eta$-strongly monotone and $\kappa$-Lipschitzian on $X$. If $\mu \in\left(0, \eta / \sigma^{2}\right)$, where $\sigma=\sqrt{\beta}(\kappa+2)$, then

$$
\psi(x)=I(x)-\mu(F+I-T)(x)
$$

is a contraction on $X$.

With the new imposed conditions and considering the above lemmas, the following corrections should be done in [1]:

(1) in Theorem 3.2 and Theorem $4.2, \mu \in\left(0, \eta / \beta k^{2}\right)$;

(2) in Theorem 5.2, $\mu \in\left(0, \eta /\left(\sigma^{2}+1\right)\right)$, where $\sigma=\sqrt{\beta}(\kappa+2)$;

(3) in Remark 5.3, $\mu \in\left(0,2(\eta-1) /\left(2 \sigma^{2}-1\right)\right)$, where $\sigma=\sqrt{\beta}(\kappa+2)$.

Also in [1, Corollary 4.3] the real Banach space $X$ does not necessarily need to have a uniformly Gateaux differentiable norm.

To avoid any ambiguity in terminology note also that $\eta$-strongly monotone mappings in Banach spaces are usually called $\eta$-strongly accretive.

\section{References}

[1] I. Mohamadi, "Iterative methods for variational inequalities over the intersection of the fixed points set of a nonexpansive semigroup in Banach spaces," Fixed Point Theory and Applications, vol. 2011, Article ID 620284, 17 pages, 2011.

[2] H. K. Xu, "Inequalities in Banach spaces with applications," Nonlinear Analysis: Theory, Methods $\mathcal{E}$ Applications, vol. 16, no. 12, pp. 1127-1138, 1991.

[3] D. J. Downing, "Surjectivity results for $\phi$-accretive set-valued mappings," Pacific Journal of Mathematics, vol. 77 , no. 2, pp. 381-388, 1978. 